\title{
Mandibular cementoblastoma: Case report
}

\author{
Cintia Mussi Milani ${ }^{1 *}$, César Augusto Thomée ${ }^{2}$ Rosangela Sayuri Saga Kamikawa ${ }^{3}$, \\ Mariana Daniel da Silva ${ }^{4}$, Maria Angela Naval Machado ${ }^{5}$ \\ ${ }^{1}$ Universidade Tuiuti do Paraná, Curitiba, Brazil \\ ${ }^{2}$ Center for Health and Biological Sciences, Pontifícia Universidade Católica do Paraná, Curitiba, Brazil \\ ${ }^{3}$ Associação Brasileira de Odontologia-Seção Paraná, Curitiba, Brazil \\ ${ }^{4}$ Private Practice, Curitiba, Brazil \\ ${ }^{5}$ Universidade Federal do Paraná, Curitiba, Brazil \\ Email: ${ }^{*}$ cintiammilani@hotmail.com
}

Received 28 November 2011; revised 12 January 2012; accepted 9 February 2012

\begin{abstract}
Cementoblastoma is a rare benign lesion that represents less than $1 \%$ of all odontogenic tumours. It's characterized by proliferation of cementum-like tissue and in almost all cases tends to be associated with an erupted permanent tooth, most often the first molar. We present an unusual case of a large cementoblastoma that affected the right mandibular body, extending from the first premolar to the second molar, of a 19-years-old male. In this case an initial surgery was attempted under local anaesthesia, resulting in incomplete tumor removal. A second surgical procedure was performed under general anaesthesia, ensuring the complete excision of the lesion. The patient was monitored for 1 year after surgery and did not show any signs of recurrence.
\end{abstract}

Keywords: Odontogenic Tumor; Cementoblasts; Oral Surgery

\section{INTRODUCTION}

Cementoblastoma is currently ranked by the World Health Organization as an odontogenic mesenchyme or ectomesenchyme tumor, with or without involvement of the odontogenic epithelium [1]. It is a benign tumor that is characterized by proliferation cementum-like tissue that is attached to the roots of teeth [2,3-6].

A cementoblastoma lesion is usually found in the mandible, most commonly in the pre-molar and molar region $[7,8]$. Its prevalence is higher in young male adults who are under 30 years of age [9]. Cementoblastomas are slow growing lesions [8-10] and are usually asymptomatic; however pain, and swelling may occur [2,3,7-15].

Radiographically, the lesion presents as a radiopaque

"Corresponding author. mass that is attached to the root tooth, which is usually vital and is surrounded by a radiolucent halo $[1,8-10,13$, 16]. Histologically, cementoblastoma tissue is similar to cementum.

The clinical and radiological findings are of great importance for the differential diagnosis of odontogenic neoplasms [8], which must include osteoblastoma, osteosarcoma and focal sclerosing osteomyelitis $[8,15]$.

The recommended treatment consists of the surgical removal of the tumor and associated teeth [2]. In cases in which an early diagnosis is made, the lesion can be removed in association with the endodontic treatment of the involved tooth [17]. If the lesion is not completely removed $[2,10]$, recurrence and continued growth can be observed due to its unlimited growth potential $[2,8,17]$, but this is not common $[2,8,10]$.

We present an unusual case of a residual cementoblastoma involving the right mandibular body of a 19years-old male.

\section{CASE REPORT}

A 19-year-old, healthy Brazilian man, was referred to a maxillofacial surgeon due to the presence of a cementoblastoma in the right mandibular body. The patient reported that he was being treated at another clinic, and his initial complaint was related to a progressive facial asymmetry.

According to the patient, the surgeon who initially treated him requested a panoramic radiograph (Figure 1) and a cone-beam tomography (Figure 2) and performed a biopsy, that led to the diagnosis of cementoblastoma. Surgery to remove the entire lesion was performed under local anesthesia, resulting in severe intraoperative pain and incomplete removal of the lesion. This initial surgery was performed three months prior to his presentation.

On clinical examination, the patient presented a painless hard swelling in the right lower posterior buccal and lingual gingiva, extending from the region of the 


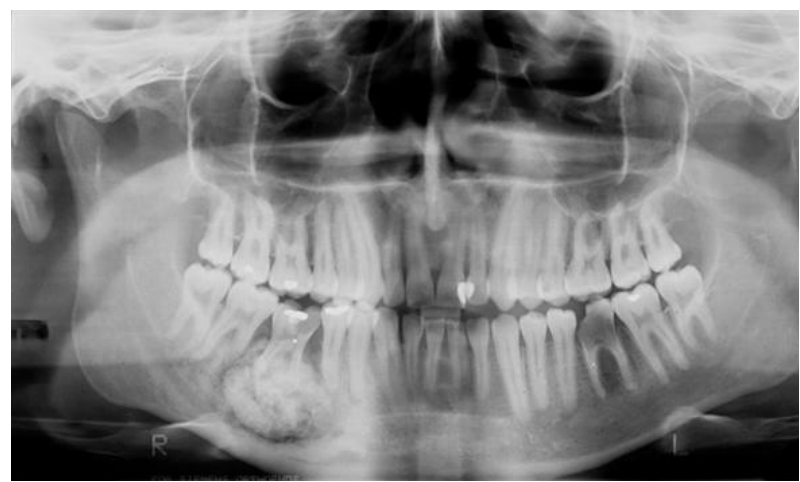

Figure 1. Panoramic radiograph showing a radiopaque lesion that is surrounded by a radiolucent halo and is in contact with the roots of the mandibular second premolar and the first and second molars.

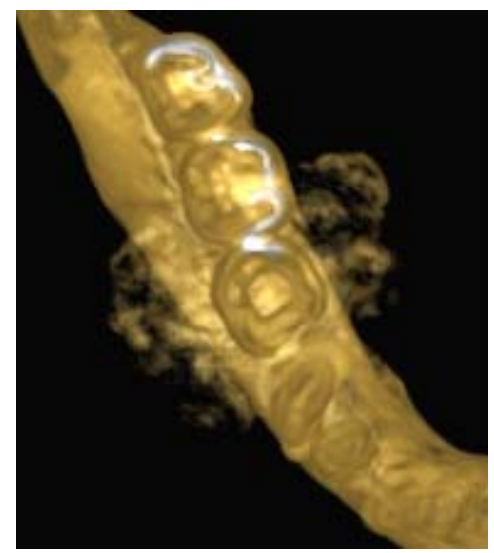

Figure 2. Cone-beam tomography showing the buccal and lingual extension of the lesion.

second premolar to the second molar on the right. These teeth were removed during the first surgery.

The initial panoramic radiograph revealed a well-defined, round, radiopaque lesion, that was delineated by a thin radiolucent halo, and was in contact with the roots of the second premolar, and the first and second right lower molars. The radiographic appearance was pathognomonic of cementoblastoma. Tomography showed the buccal and lingual extension of the lesion. The panoramic radiograph performed after the first surgery revealed that a large amount of the lesion was left in place, and a fracture in the apical portion of the mesial root of the third molar occurred intraoperatively (Figure 3). A vitality test of the third molar revealed that this tooth was still vital.

Based on clinical and radiological findings, surgery under general anesthesia was planned to remove the remainder of the lesion. A subsequent reconstructive surgery was also planned to reconstruct the area with bone grafts and posteriorly osseointegrated implants.

Surgical removal of the residual tumor was performed at a hospital under general anesthesia. A buccolingual mucoperiosteal flap, extending from the lower right canine to the lower right third molar, was raised, and an osteotomy was performed to separate the remaining tumor from the subjacent bone with complete curettage of the cavity (Figure 4). Histopathological examination confirmed the initial diagnosis of cementoblastoma. Microscopic examination revealed a cementum-like calcified tissue with dense trabeculae of mineralized material. The cement was intensely basophilic and exhibited irregular reversal lines (Figure 5). The presence of fibrovascular tissue cells dispersed throughout the mineralized material or at the periphery of the lesion was extensive as well as the presence of multinucleated giant cells.

In the postoperative period, the patient presented inferior alveolar nerve paresthesia, which resolved spontaneously 20 days later. Panoramic radiography performed immediately after surgery revealed complete tumor removal. The patient was monitored for 1 year after surgery, and did not show any signs of recurrence (Figure 6). After this period, he was lost upon follow-up, and the reconstruction that was originally planned was not possible.

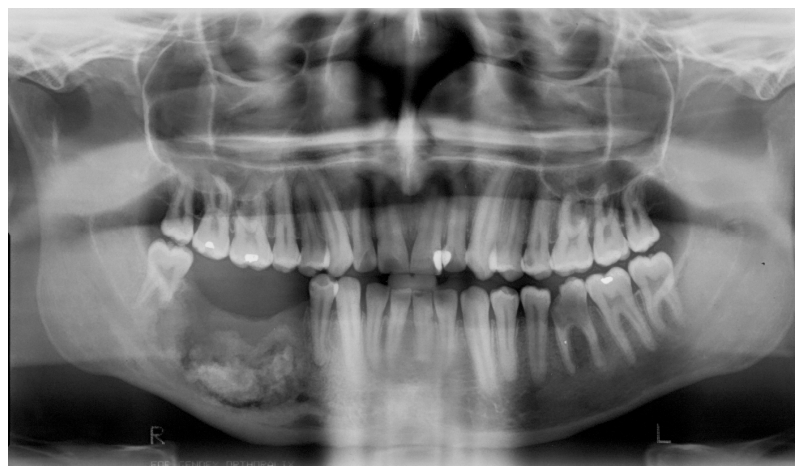

Figure 3. Postoperative panoramic radiograph after the initial surgery showing large amounts of residual lesions and a fracture of the mesial root of the lower right third molar.

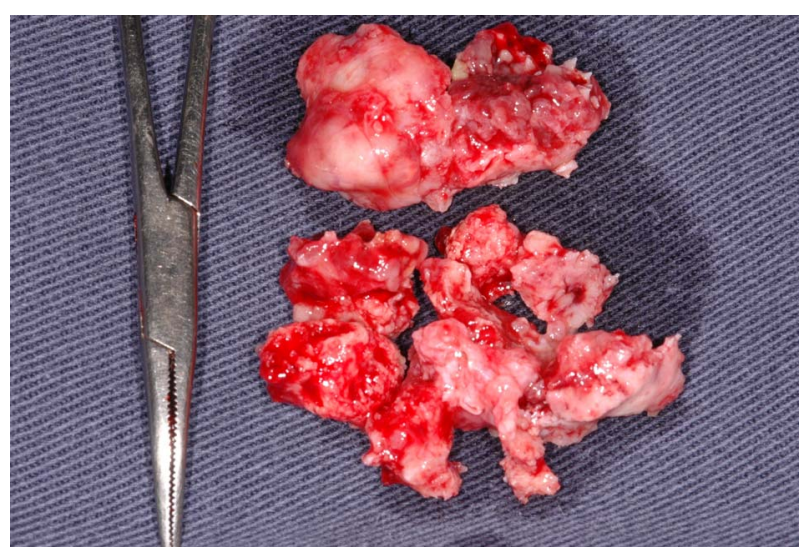

Figure 4. Residual tumor removed in the second surgery. 


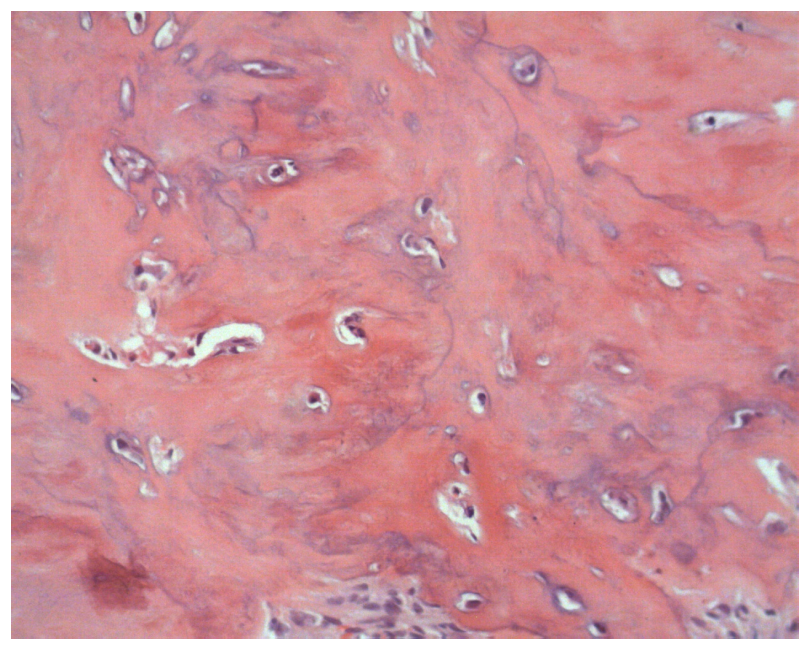

Figure 5. Histologic findings: Osteocementous calcified tissue with thick trabeculae of mineralized material containing numerous gaps and cementocytes. (H.E. magnification 20×).

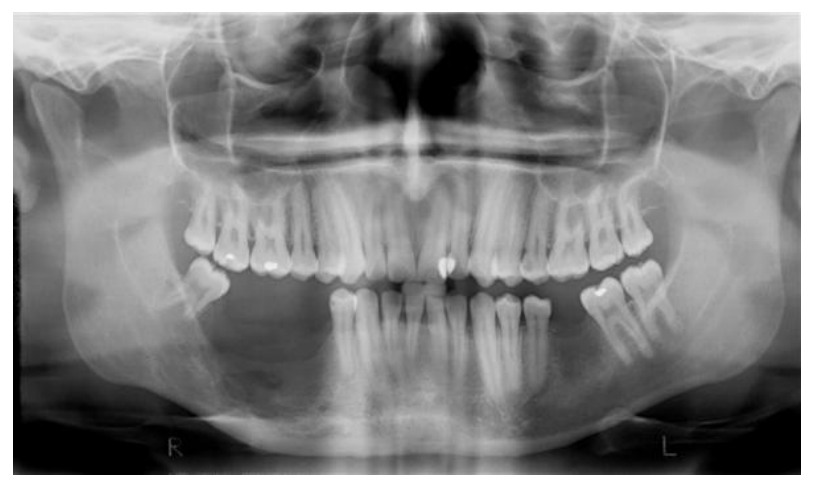

Figure 6. Panoramic radiograph at the one year follow-up. Local bone remodeling and no signs of recurrence.

\section{DISCUSSION}

Cementoblastoma is a rare odontogenic neoplasm, with an estimated occurrence, according to Baart et al. [14], of less than 1 case per million people per year. Young adults who are under 30 years of age are most commonly affected [1,3,7-15,17], with a slight male prevalence [2,3,9].

According to Brannon et al. [2], 79.5\% of tumors occur in the mandible, and in most cases, the lesion involves the first permanent molar $[1,7,12,14,17]$; in the few cases in which the upper jaw is affected, the maxillary sinus can be involved $[3,13]$. There are also reports of cementoblastoma associated with impacted $[8,11,13]$ and deciduous teeth $[3,10]$. The occurrence of the lesion involving more than one permanent tooth, as in the case reported here, is rare [3,12-14]. Regardless of the number of teeth involved, the lesion is always associated with the dental root [1-18].

Patients may be completely asymptomatic $[1,2,8]$; however, bone expansion and pain can occur [2,3,7-15]. Trismus $[8,10]$, dental displacement, and increased mo- bility of adjacent teeth [14] may eventually be observed.

Radiographically, the lesion presents as a well-defined radiopaque mass, surrounded by a radiolucent halo associated with the root of the tooth or teeth $[1,3,7-12,14,15$, 17,18]. Depending on the developmental stage of the lesion, the radiological appearance and clinical interpretation can vary, leading to an erroneous diagnosis of osteoma or osteoblastoma $[8,13,14]$. Other features that can eventually be observed on radiographs include root resorption $[1-3,10,14,17,18]$, obliteration of the periodontal ligament space [2,7], and invasion of the root canals [3].

Histologically, cementoblastoma is characterized by tissue layers that are similar to cement $[3,13]$ and, consist of poorly mineralized cellular material with prominent basophilic reversal lines $[1-3,15,17,18]$ organized in a fibrovascular stroma [1-3,9,12,13,18]. Prominent cementoblasts [12], irregular lacunae [3,8,15], increased activity of cementoblasts and cementoclasts [2], and trabeculae of uncalcified matrix perpendicular to the surface $[1,7,12,14,18]$ are typical characteristics of this lesion.

The most difficult challenge in the differential diagnosis of cementoblastoma is osteoblastoma. These two tumors may exhibit the same histomorphology [18], but they differ in their origin [2]. Some authors defend the odontogenic origin as a basis to differentiating cementoblastoma from osteoblastoma; this is because in cementoblastoma, the lesion is part of the root structure of the involved tooth caused by neoplastic cementoblasts that produce mineralized material that fuses with a tooth root structure $[7,17,18]$. Confirming this, Cundiff [7] presented a case in which the growth of a cementoblastoma was radiographically followed for more than four years. He described rare findings, such as a slight enlargement of the periodontal ligament space, until the complete removal of the tumor when it was $3 \mathrm{~cm}$ in diameter. Osteoblastomas are tippically separated from the adjacent tooth by a barrier that is formed by the periodontal ligament [18]. Columns of tissue that are similar to nonmineralized cementum that are located on the periphery of the mass also support the presence of a cementoblastoma [14]. Both cementoblastomas and osteoblastomas may cause root resorption [18].

In addition to osteoblastoma, the differential diagnosis of cementoblastoma must also include osteosarcoma [1], osteoma [8], focal sclerosing osteomyelitis, osteoesclerosis, and fibrous dysplasia [12,13].

The recommended treatment of cementoblastomas consists of the surgical removal of the lesion along with the tooth/teeth and/or structures that are affected, followed by complete curettage of the area or the peripheral osteotomy of the entire region $[2,8,9,10,14]$. When an early diagnosis is made, the treatment may involve the complete excision of the lesion with preservation of the involved tooth, thorough endodontic treatment [17] and, 
in some cases, apicoectomy [15]. For those cases in which a late diagnosis is made and the tumor has already achieved major proportions, as in this report, the complete removal of the lesion and associated structures is recommended due to the unlimited growth potential $[8,17]$ and eventual recurrence. In these cases, the surgical procedure must be performed under general anesthesia $[2,3,11,12,14]$, which ensures a less stressful intraoperative time for the patient and for the surgical team. As we observed in the present case, the initial surgery under local anesthesia resulted in surgical failure because the surgeon was not able to achieve the final objective of the complete removal of the lesion. In addition, the patient was exposed to an unnecessary psychological trauma.

Recurrences are rare if complete tumor enucleation is performed. Brannon et al. [2], stated that recurrence is more common when curettage is performed without the extraction of the involved tooth or teeth. Mandibular expansion and perforation of the cortex are clinical signs of recurrence [2,9]. Studies indicate recurrence between 6 months and 1 year after the initial surgery $[2,9,14]$.

\section{CONCLUSION}

Cementoblastoma is a benign tumor with a low recurrence rate but unlimited growth potential. Appropriate treatment consists of the surgical removal, and early diagnosis favors a more conservative surgery with the possibility of preservation of the involved teeth. In cases in which the tumor is detected in advanced stages of development, the teeth should be removed along with the tumor to decrease the possibility of recurrence.

\section{REFERENCES}

[1] Hubber, A.R. and Folk, G.S. (2009) Cementoblastoma. Head and Neck Pathol, 3, 133-135. doi:10.1007/s12105-008-0099-5

[2] Brannon, R.B., Fowler, C.B., Carpenter, W.M. and Corio, R.L. (2002) Cementoblastoma: An innocuous neoplasm? A clinicopathologic study of 44 cases and review of literature with special emphasis on recurrence. Oral Surgery, Oral Medicine, Oral Pathology, Oral Radiology, and Endodontology, 93, 311-320. doi:10.1067/moe.2002.121993

[3] Ohki, K., Kumamoto, H., Nitta, Y., Nagasaka, H., Kawamura, H. and Ooya, K. (2004) Benign cementoblastoma involving multiple maxillary teeth: Report of a case with a review of the literature. Oral Surgery, Oral Medicine, Oral Pathology, Oral Radiology, and Endodontology, 97, 53-58. doi:10.1016/j.tripleo.2003.08.012

[4] Barnes, L., Eveson, J.W., Reichart, P. and Sidransky, D. (2005) World Health Organization Classification of tumours: Pathology and genetics of tumours of the head and neck. IARC Press, Lyon.

[5] Lee, Y., Xuan, M., Takata, T., Wang, C., He, Z., Zhou, Z., Mock, D. and Nickai, H. (1998) Odontogenic tumors. A demographic study of 759 cases in a chinese population. Oral Surgery, Oral Medicine, Oral Pathology, Oral Radiology, and Endodontology, 86, 707-714.

[6] Ochsenius, G., Ortega, A., Godoy, L., Penafieli C. and Escobar, E. (2002) Odontogenic Tumors in Chile: A study of 362 cases. Journal of Oral Pathology \& Medicine, 31, 415-420. doi:10.1034/j.1600-0714.2002.00073.x

[7] Cundiff, E.J. (2000) Developing cementoblastoma: Case report and update of differential diagnosis. Quintessence International, 31, 191-195.

[8] Sumer, M., Gunduz, K., Sumer, P.A. and Gunhan, O. (2006) Benign cementoblastoma: A case report. Medicina Oral, Patología Oral Y Cirugía Bucal, 11, 483-485.

[9] Zaitoun, H., Kujan, O. and Sloan, P. (2007) An unusual recurrent cementoblastoma associated with a developing lower second molar tooth: A case report. Journal of Oral and Maxillofacial Surgery, 65, 2080-2082. doi:10.1016/j.joms.2006.06.288

[10] Vieira, A.P.G.F., Meneses, J.M.S. Jr. and Maia, R.L. (2007) Cementoblastoma related to a primary tooth: A case report. Journal of Oral Pathology \& Medicine, 36, 117-119. doi:10.1111/j.1600-0714.2007.00465.x

[11] Piattelli, A., Di Alberti, L., Scarano, A. and Piatelli, M. (1998) Benign cementoblastoma associated with an unerupted third molar. Oral Oncology, 34, 229-231.

[12] Pontes, F.S.C., Carneiro, J.T. Jr., Ribeiro, A.L.R., Gonçalves, A. Jr., Fonseca, F.P., Pontes, H.A.R. and Pinto, D.S. Jr. (2008) Cementoblastoma previously misdiagnosed as fibrous dysplasia: Report of an uncommon case and discussion of the differential diagnosis. International Journal of Pediatric Otorhinolaryngology Extra, 3, 182-187. doi:10.1016/j.pedex.2008.04.001

[13] Infante-Cossio, P., Hernandez-Guisado, J.M., Acosta-Feria, M. and Carranza-Carranza, A. (2008) Cementoblastoma involveing the maxillary sinus. British Journal of Oral and Maxillofacial Surgery, 46, 234-236. doi:10.1016/j.bjoms.2007.03.009

[14] Baart, J.A., Lekkas, C. and Van der Waal, I. (1991) Residual cementoblastoma of the mandible. Journal of Oral Pathology \& Medicine, 20, 300-302. doi:10.1111/j.1600-0714.1991.tb00932.x

[15] Hirai, E., Yamamoto, K., Kounoe, T., Kondo, Y., Yonemasu, H. and Kurokawa, H. (2010) Benign cementoblastoma of the anterior maxilla. Journal of Oral and Maxillofacial Surgery, 68, 671-674. doi:10.1016/j.joms.2009.03.060

[16] Ulmanski, M., Hansen, E., Praetorius, F. and Haque, M.F. (1994) Benign Cementoblastoma: A review and five new cases. Oral Surgery, Oral Medicine, Oral Pathology, 77, 48-55. doi:10.1016/S0030-4220(06)80106-4

[17] Biggs, J.T. and Benenati, F.W. (1995) Surgically treating a benign cementoblastoma while retaining the involved tooth. Journal of the American Dental Association, 126, 1288-1290.

[18] Slootweg, P.J. (1992) Cementoblastoma and osteoblastoma: A comparison of histologic features. Journal of Oral Pathology \& Medicine, 21, 385-389. doi:10.1111/j.1600-0714.1992.tb01024.X 